at page 272 of the B.M.f. this week. They have measured histamine released quantitatively from chopped human lung passively sensitized by reagin-containing serum and reacted with the relevant allergen. Fresh human lung is required, because human reaginic antibody reacts only with human or primate tissues. The quantitative assay of reagin in this method is, as the authors indicate, an indirect one, and its biological standardization still needs to be worked out.

In patients with clinical allergy and positive skin tests to grass pollen, substantial histamine release was detected in all except one. The exceptional patient is important, because positive skin tests and histamine release from her own leucocytes had indicated sensitization of her tissues. Thus measurement of circulating reaginic antibody by this passive sensitization technique does not always reflect the degree of active tissue sensitization. It will be important to know whether reaginic titres measured by this method correlate with the clinical severity of the allergy and with desensitization or other forms of therapy.

This method may be useful for detecting reaginic antibody to antigens which may be undesirable to use in skin tests-for example, some industrial agents. It could also be used to study intrinsic asthma. Not only could a wide range of antigens be tested, but negative results might provide additional evidence in support of other work ${ }^{5}$ that intrinsic asthma does not depend on reagins. It could also be used to establish or exclude the role of reagins in causing asthmatic attacks developing after an interval of some hours following exposure to such antigens as avian proteins ${ }^{6}$ and those causing farmer's lung. ${ }^{78}$

In the second part of their paper Assem and Schild report their results with sera from patients with penicillin allergy. Once again drug hypersensitivity has proved a refractory problem. Only three out of eight sera from patients with established sensitivity gave positive results by the passive sensitization test, usually with only low levels of histamine release. The correlation between these in-vitro studies and the skin tests was also poor. These discouraging results may in part be due to our incomplete knowledge of the molecular structure of the complete antigen and also perhaps because penicillin allergy may also depend on non-reaginic mechanisms; certainly other types of circulating antibody have also been detected in these patients. ${ }^{8}$

There is an urgent need for an in-vitro test for drug allergy, because skin testing is known to be unreliable and sometimes dangerous. It is disappointing that the technique described here does not fill this need. The sequence of tests suggested in this paper to exclude the majority of severe penicillin allergies are both time-consuming and dependent on a ready supply of fresh human or monkey lung; they are therefore at best suitable only when no urgent therapeutic action has to be taken. Unfortunately, as the authors state, complete safety cannot be ensured by any test at present available, so for the time being the best practical solution is to avoid penicillin if there is a suggestive history of hypersensitivity.

1 Shelley, W. B., J. Amer. med. Ass., 1963, 184, 171.

'Ridges, A. P., and Augustin, R., Nature (Lond.), 1964, 202, 667.

' Ishizaka, K., and Ishizaka, T., F. Immunol., 1967, 99, 1187.

- Johansson, S. G. O., Bennich, H., and Wide, L., Immunology, 1968, 14, 265.

"Johansson, S. G. O., Lancet, 1967, $2,951$.

- Pepys, J., Hargreave, F. E., Chan, M., and McCarthy, D. S., Lancet, $1968,2,134$.

' Pepys, J., f. roy. Coll. Phycns Lond., 1967, 2, 42.

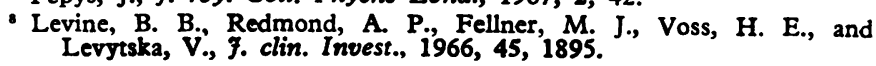

\section{Neurology of Depression}

The successful treatment of much mental depressive illness with drugs implies some kind of physical disorder in the patient's brain. But what this is remains obscure, partly because conventional diagnostic neurology concentrates on reflexes and motor and perceptual tests, ignoring other aspects of central control. Yet there are already some clinical hints of what to look at in the depressed patient. Vegetative symptoms such as anorexia and loss of weight, diminished salivation, constipation, and insomnia are all part of the picture of depressive illness, even if often overshadowed by disturbance of mood or depressive ideation. Vegetative control may be partly hypothalamic, and thence partly exerted through the anterior pituitary and its various hormones, of which corticotrophin (A.C.T.H.) and its stimulation of the secretion and release of cortisol are the best-worked-out mechanisms.

Emotional excitement such as examinations, for instance, can cause a rise in the level of cortisol in the plasma, but more important at present are two other facts. One is that the diurnal variation in the plasma level of cortisol, with a maximum around 8 a.m. every day and a minimum near midnight, is a rhythm dictated by the central nervous system, and changes in its timing may result from lesions in certain areas of the brain. ${ }^{1}$ The second is that the level of plasma cortisol controls the rate at which corticotrophin is released; this is effected through an action of cortisol on the central nervous control of the pituitary and not directly on the gland itself. A sudden rise in plasma cortisol results in a reduced release of corticotrophin and hence a less active adrenal cortex and less secretion of cortisol, and so correction of the level.

Dexamethasone is a synthetic glucocorticoid related to prednisolone. It is not estimated by methods which measure plasma cortisol, yet it acts like cortisol on the nervous system in preventing release of corticotrophin. Consequently, giving dexamethasone lowers the plasma cortisol, and measuring this response tests the functional integrity of the hypothalamo= pituitary-adrenal control of the secretion of cortisol. Dr. B. J. Carroll, Dr. F. I. R. Martin, and Professor Brian Davies in a paper from Melbourne (see page 285) report on the application of this test to severely depressed psychiatric inpatients. They show that many depressives have become unresponsive but recover normal responsiveness when they return to a normal mental state. Thus while they are ill the hypothalamic control is escaping from its normal steroid restraint and corticotrophin continues to be liberated even when the plasma level of steroid rises, until a new higher equilibrium is reached.

This finding agrees with that made by P. W. P. Butler and G. M. Besser ${ }^{2}$ at St. Bartholomew's Hospital. These workers also observed some disturbance of diurnal rhythm during the depression in their three cases, while others ${ }^{3}$ have reported an upset of the diurnal variation which became normal when the patients recovered. There is thus a growing body of evidence that higher nervous control of the pituitary may be altered in depressive illness. The hypothalamic response to intravenous insulin, resulting in secretion of

\footnotetext{
Krieger, D. T., and Krieger, H. P., F. clin Endocrin, 1966, 26, 929.

2 Butler, P. W. P., and Besser, G. M.,'Lancet, 1968, 1, 1234.

Doig, R. J., Mummery, R. V., Wills, M. R., and Elkes, A., Brit. 7 . Psychiat., 1966, 112, 1263.

Greenwood, F. C., Landon, J., and Stamp, T. C. B., F. clin. Invest. $1966,45,429$

s Landon, J., et al., f. clin. Invest., 1966, 45, 437.
} 
growth hormone as well as corticotrophin, has also been developed into a neurological test, ${ }^{45}$ and Carroll has given preliminary results to an Australian symposium to show that there is likewise an increased degree of insulin resistance (strictly, insensitivity to hypoglycaemia) to be measured during depressive illness.

One of the questions in psychiatry today is how to break down the mass of depressive illnesses into subgroups, each with an effective drug treatment and known prognosis. Functional neurology, using tests like the dexamethasone, may help to establish these useful clinical divisions. But a second function of this work is to focus greater attention on the neural control of the pituitary and its modification by drugs, a field of animal research which the psychopharmacologist may decide requires thorough analysis.

\section{Bacterial Interference in the Nursery}

A newborn infant faces an immediate onslaught by many bacteria, including potential pathogens. Much has been done to protect it against one of these, Staphylococcus pyogenes, by the use of hexachlorophane (U.S.P., hexachlorophene), an antiseptic with a persistent and cumulative action. There is ample evidence that its use will greatly reduce the frequency of colonization of the nose and umbilical stump by pathogenic staphylococci, with a consequent reduction in the occurrence of septic lesions in the infant and sometimes of breast abscesses in the mothers.

A study ${ }^{1}$ of the effects of using this and other antiseptics in the infant nurseries at the Cincinnati General Hospital brings out some new points, particularly with regard to the effect of these measures on other bacteria than staphylococci. These effects were judged solely by examining the flora of the nose and umbilical stump ; there is no mention of any septic lesions. Three procedures were successively introduced. The first was simply hexachlorophane hand washing by the staff. During this period the frequency of colonization of nose and umbilicus by pathogenic staphylococci was 54 and $50 \%$. When, secondly, daily bathing of the infants in addition was introduced with a preparation containing hexachlorophane and a detergent (Phisohex) these figures fell to 5.5 and $4 \%$, but there was an increase in the frequency of colonization of the umbilical stump by coliform bacilli from 45 to $78 \%$ and a corresponding smaller increase in the nose. Similar observations were recently reported by J. O. Forfar and colleagues. $^{2}$ Finally, apparently in an attempt to counter this change, staff hand washing was followed by the application of an antibacterial foam containing $50 \%$ alcohol and $0.25 \%$ of each of two quaternary ammonium compounds said to possess "modest activity" against some Gram-negative organisms. This was followed by an equally modest reduction in the occurrence of coliform bacilli in the nose, but none at the umbilical site.

i Light, I. J., Sutherland, J. M., Cochran, M. L., and Sutorius, J., New Engl. F. Med., 1968, 278, 1243.

'Forfar, J. O., Gould, J. C., and Maccabe, A. F., Lancet, 1968, 2, 177.

" Hays, E. E., et al., \$. biol. Chem., 1945, $159,725$.

"Shinefield, H. R., Ribble, J. C., Eichenwald, H. F., Boris, M., and Sutherland, J. M., Amer. F. Dis. Child., 1963, 105, 683 .

" Light, I. J., Sutherland, J. M., and Schott, J. E., F. Amer. med. Ass., $1965,193,699$.

- Light, I. J., Walton, R. L., Sutherland, J. M., Shinefield, H. R., and Brackvogel, V., Amer. 尹. Dis. Child., 1967, 113, 291.
It seems doubtful whether the effect of this last procedure was significant, and the interest of the findings lies rather in the relationship between the two forms of colonization. Of the four varieties of coliform bacillus distinguished, Escherichia coli, Proteus, and Klebsiella-Aerobacter were often found when staphylococci were also present, but Pseudomonas (not distinguished as to species), though isolated from 156 out of 4,527 swabs, was accompanied by Staph. aureus only once, in an infant with purulent rhinitis. The authors offer no explanation of the mechanism of this apparent antagonism. It is in fact readily explicable if their organisms were Ps. aeruginosa (pyocyanea). Pyocyanase (should we now call this " aeruginase" ?), a product of this organism, was for many years used as an antiseptic, particularly on the Continent, and staphylococci are among the organisms most susceptible to its action. ${ }^{3}$

The subtitle of the Cincinnati paper is "Another Example of Bacterial Interference," which is true enough, but not of practical interest, since no one would venture deliberately to plant Ps. aeruginosa in an infant for the sake of keeping out staphylococci. But an example of bacterial interference with which the authors compare this has been turned to practical account by themselves and others. ${ }^{4-6}$ This is the introduction into the nose and umbilical stump shortly after birth of small numbers of a strain of coagulase-positive staphylococcus known as $502 \mathrm{~A}$, which appears from its record to be nonpathogenic. Successful colonization usually follows, and occupation of these sites by this organism repels invasion by another virulent strain during an epidemic. The evidence that such protection can be achieved seems conclusive from these papers, for they include large reductions in the incidence of septic lesions as compared with controls. Ethical objections have been raised to this procedure, but it seems no more objectionable from this standpoint than the use of living vaccines. It may indeed be asked whether the colonization of other parts of the infant's body should be left entirely to chance, and there are many precedents for the implantation of supposedly beneficent bacteria in the alimentary tract.

\section{Renal Damage in Chicken-pox}

Acute glomerulonephritis is traditionally associated with streptococcal infections, but evidence is accumulating that other organisms may also cause it. With better laboratory techniques viruria has been detected in such diverse infections as mumps, ${ }^{1}$ enterovirus, ${ }^{3-5}$ rubella, ${ }^{6}{ }^{7}$ measles, ${ }^{8}$ cytomegalovirus, ${ }^{9}$ adenovirus, ${ }^{1011}$ and vaccinia, ${ }^{10}$ to name but a few. Clinical and histological evidence of renal damage in virus diseases suggests that viruria results from active infection of kidney cells rather than passive filtration of virus from the blood stream.

In recent years acute glomerulonephritis has been reported during the course of mumps, ${ }^{212}$ infectious hepatitis, ${ }^{13}$ E.C.H.O. ${ }^{14}{ }^{15}$ and adenovirus ${ }^{16}$ infections, and chickenpox. ${ }^{17-22}$ Transient minor disturbance of renal function, as shown by creatinine-clearance tests, is particularly common in mumps ${ }^{2}$ and may occasionally progress to florid nephritis with a fatal issue. In 20 patients suffering from infectious hepatitis M. E. Conrad and others ${ }^{13}$ discovered 10 with transient proteinuria and 8 with haematuria. Renal biopsy 